\title{
miRFANs: an integrated database for Arabidopsis thaliana microRNA function annotations
}

Hui Liu ${ }^{1}$, Ting Jin ${ }^{2,5}$, Ruiqi Liao², Linxia Wan², Bin Xu², Shuigeng Zhou ${ }^{2,4^{*}}$ and Jihong Guan ${ }^{3 *}$

\begin{abstract}
Background: Plant microRNAs (miRNAs) have been revealed to play important roles in developmental control, hormone secretion, cell differentiation and proliferation, and response to environmental stresses. However, our knowledge about the regulatory mechanisms and functions of miRNAs remains very limited. The main difficulties lie in two aspects. On one hand, the number of experimentally validated miRNA targets is very limited and the predicted targets often include many false positives, which constrains us to reveal the functions of miRNAs. On the other hand, the regulation of miRNAs is known to be spatio-temporally specific, which increases the difficulty for us to understand the regulatory mechanisms of miRNAs.
\end{abstract}

Description: In this paper we present miRFANs, an online database for Arabidopsis thaliana miRNA function annotations. We integrated various type of datasets, including miRNA-target interactions, transcription factor (TF) and their targets, expression profiles, genomic annotations and pathways, into a comprehensive database, and developed various statistical and mining tools, together with a user-friendly web interface. For each miRNA target predicted by psRNATarget, TargetAlign and UEA target-finder, or recorded in TarBase and miRTarBase, the effect of its up-regulated or down-regulated miRNA on the expression level of the target gene is evaluated by carrying out differential expression analysis of both miRNA and targets expression profiles acquired under the same (or similar) experimental condition and in the same tissue. Moreover, each miRNA target is associated with gene ontology and pathway terms, together with the target site information and regulating miRNAs predicted by different computational methods. These associated terms may provide valuable insight for the functions of each miRNA.

Conclusion: First, a comprehensive collection of miRNA targets for Arabidopsis thaliana provides valuable information about the functions of plant miRNAs. Second, a highly informative miRNA-mediated genetic regulatory network is extracted from our integrative database. Third, a set of statistical and mining tools is equipped for analyzing and mining the database. And fourth, a user-friendly web interface is developed to facilitate the browsing and analysis of the collected data.

\section{Background}

miRNAs are a class of short ( $\sim 22$ nucleotide) noncoding RNAs expressed in both animal and plant cells. These small RNA molecules bind preferably to the 3' untranslated region ( $\left.3^{\prime} \mathrm{UTR}\right)$ of protein coding genes and make them degradation and/or translation inhibition [1]. A typical miRNA target site has perfect or

\footnotetext{
*Correspondence: sgzhou@fudan.edu.cn; jhguan@tongji.edu.cn

${ }^{2}$ School of Computer Science, Fudan university, Shanghai, China

${ }^{3}$ Department of Computer Science \& Technology, Tongji University, Shanghai, China

Full list of author information is available at the end of the article
}

nearly perfect complementarity to the miRNA so-called seed sequence, i.e., $\sim 7$ nucleotides from miRNA 5 ' end $[2,3]$. Both experimental and computational studies have shown that miRNAs are a class of post-transcriptional regulators, and play important roles in cellular process $[4,5]$, developmental control [6,7], hormone secretion [8], cell differentiation and proliferation [9], and responses to environmental stresses $[10,11]$. One challenge facing miRNA research is to accurately identify the target mRNAs, because of the very limited sequence complementarity between miRNAs and their targets, and the 
scarcity of experimentally validated gene targets to guide accurate prediction models [12].

Arabidopsis thaliana, a small flowering plant with only several weeks of life cycle, has important advantages for the research of genetics and molecular biology, thus some special databases for Arabidopsis thaliana have been established. TAIR is a comprehensive information system that deposits genome, expression profiling, proteome, pathway and functional annotations, together with many analysis and visualization tools [13]. For miRNAs, Sanger Institute's miRBase serves as the central depository where miRNAs are experimentally validated. The current release, version 10.0, contains over 266 Arabidopsis thaliana miRNAs [14]. However, the functions of most miRNAs are still unknown so far. The Arabidopsis Small RNA Project Database, abbreviated as ASRP, intends for Arabidopsis thaliana small RNA mature sequences, transcripts and locus [15]. PMRD (Plant miRNA Database) has collected miRNA sequences, miRNA target genes and expression profiles of some model plants [16]. mirEX [17] collects information about the expression profiles of 190 Arabidopsis thaliana primiRNAs in seven different developmental stages and provides simultaneous comparison of expression levels between various microRNA genes in diverse organs and developmental stages.

However, all databases mentioned above pay main attention to collecting miRNA-related information such as miRNA sequences, miRNA-target bindings. No system has been devoted to the functional exploration of miRNAs. Even though a few databases include expression information of miRNAs, the coverage is quite limited, failing to integrate most of the microarray and highthroughput experimental results. The recently published database miRNEST [18] is a comprehensive collection of animal, plant and virus microRNA-related data, including miRNA sequence, expression, polymorphisms and targets data, but it has no function annotations and pathways data, and does not provide comparative analysis of expression profiles from identical tissues or samples between miRNAs and their targets.

In this paper, we introduce miRFANs, an integrated database with a friendly web interface for functional annotations of miRNAs, which encompasses miRNAtarget interactions, expression, functional annotations, pathway terms. For miRNA targets, three predicted sets by three computational algorithms (psRNATarget [19], TargetAlign [20] and UEA target-finder [21]) and two experimentally validated sets (miRTarBase [22] and TarBase [23]) are collected. However, the miRNA-target interactions predicted by computational methods only imply the possibility that miRNAs regulate their targets under certain spatio-temporal conditions, as the regulation of miRNAs is well known to be condition- and tissue-specific. The expression data provides us the chance to evaluate the effect of miRNA binding in the sense of a certain environmental condition and tissue, on the basis of several evidences showing that the expression profiles of miRNAs are closely related to that of their target genes. The reciprocal expression patterns between miRNAs and their targets can be remarkable evidence of miRNA regulatory events. Therefore, we download expression datasets of both miRNAs and genome from the gene expression omnibus (GEO) [24]. Expression datasets are preprocessed and integrated for readily identifying co-expressed or differentially expressed mRNAs and their targets. We also developed a web interface supporting diverse query entries that include search by miRNA, expression profile or pathway, and a mining toolbox including correlation, differential expression analysis and clustering to explore the expression data.

Thus, miRFANs can serve as a comprehensive resource for exploring the functions of Arabidopsis thaliana miRNAs.

\section{Construction and content}

\section{Data sources and preprocessing}

In what follows, we introduce the data sources and preprocessing procedures for constructing the database.

\section{miRNAs and miRNA targets}

Mature miRNA sequences are downloaded from miRBase database (release 17), which includes 266 miRNAs of Arabidopsis thaliana, the whole genome sequences are obtained from TAIR.

miRNA target genes are acquired by four ways: 1) experimentally validated miRNA target genes from two manually curated databases, TarBase [23] and miRTarBase [22]. For convenience, we combine them into one dataset denoted by (miR)TarBase that includes 81 miRNA-target interactions; 2) miRNA targets predicted by psRNATarget [19], which uses the scoring schema originally applied by miRU [25] to evaluate the complementarity between small RNA and target gene transcript, and utilize RNAup included in Vienna Package [26] to estimate the target site accessibility; 3) miRNA targets predicted by TargetAlign [20], a Smith-Waterman-like alignment tool that uses dynamic programming to build a score matrix based on the complementarity of nucleotides for tracing the optimal local alignments; and 4) miRNA targets predicted by target-finder developed by UEA lab [21], which tries to predict miRNA target genes from high-throughout sequencing data. To the best of our knowledge, miRFANs is the most comprehensive miRNA targets database up to date. For more details of the data please see Additional File 1. 


\section{Expression profiles}

The miRNA expression profiles are obtained from highthroughout sequencing datasets downloaded from Gene Expression Omnibus (GEO), ASRP and PMRD. These datasets are normalized by using miRAnalyzer [27], which adopts a fast, short read alignment algorithm Bowtie [28] to map the short reads to mature miRNA sequences, and counts the number of mapped reads for each miRNA. miRAnalyzer outputs both the read count and normalized value for each miRNA. The normalized value is computed as read count which is first divided by the read count of all unique reads mapped to this library and then times 100. In total, the miRNA expression datasets include 12 experiments, 81 samples and 13 types of tissues.

The genome-wide expression profiles are downloaded from GEO [24], the datasets include 548 microarray experiments, 6740 samples, 53 types of tissues. For single channel data, expression levels are normalized to count data, reflecting the relative measure of abundance of each transcript. For dual channel experiments, expression levels are normalized to log ratios. For low quality or absent signals, we run KNNImpute [29] to estimate the expression levels.

\section{TFs and target genes}

As a gene may be regulated by both TFs and miRNAs, integration of the regulation relationship between TFs and their target genes into the database will give more insight into the combinatorial regulation of miRNAs and TFs to their common target genes. We download the TF and regulatory network datasets from AGRIS [30,31] website and integrate them into our database.

\section{GO and pathways}

To explore the functional annotations and pathways of miRNA targets, we download the whole genome annotations from GO [32] and pathways from KEGG [33].

We integrate all the data mentioned above by designing a new database schema, and thus obtain a comprehensive database for Arabidopsis thaliana miRNAs and their targets. The flowchart of the data source and integration process is shown in Figure 1.

\section{Comparison of differential expression}

In general, the interactions between a miRNA and its targets predicted by computational methods imply merely the possibility that the miRNA regulates the targets under a certain spatio-temporal condition, as the regulation of miRNA is well known to be condition- and tissuespecific. However, up to now we still know little about in which tissues, to what environmental stimuli and on which development stages miRNAs regulate their targets.

The major goal of miRFANs is to tackle the problem by conducting differential expression comparison between

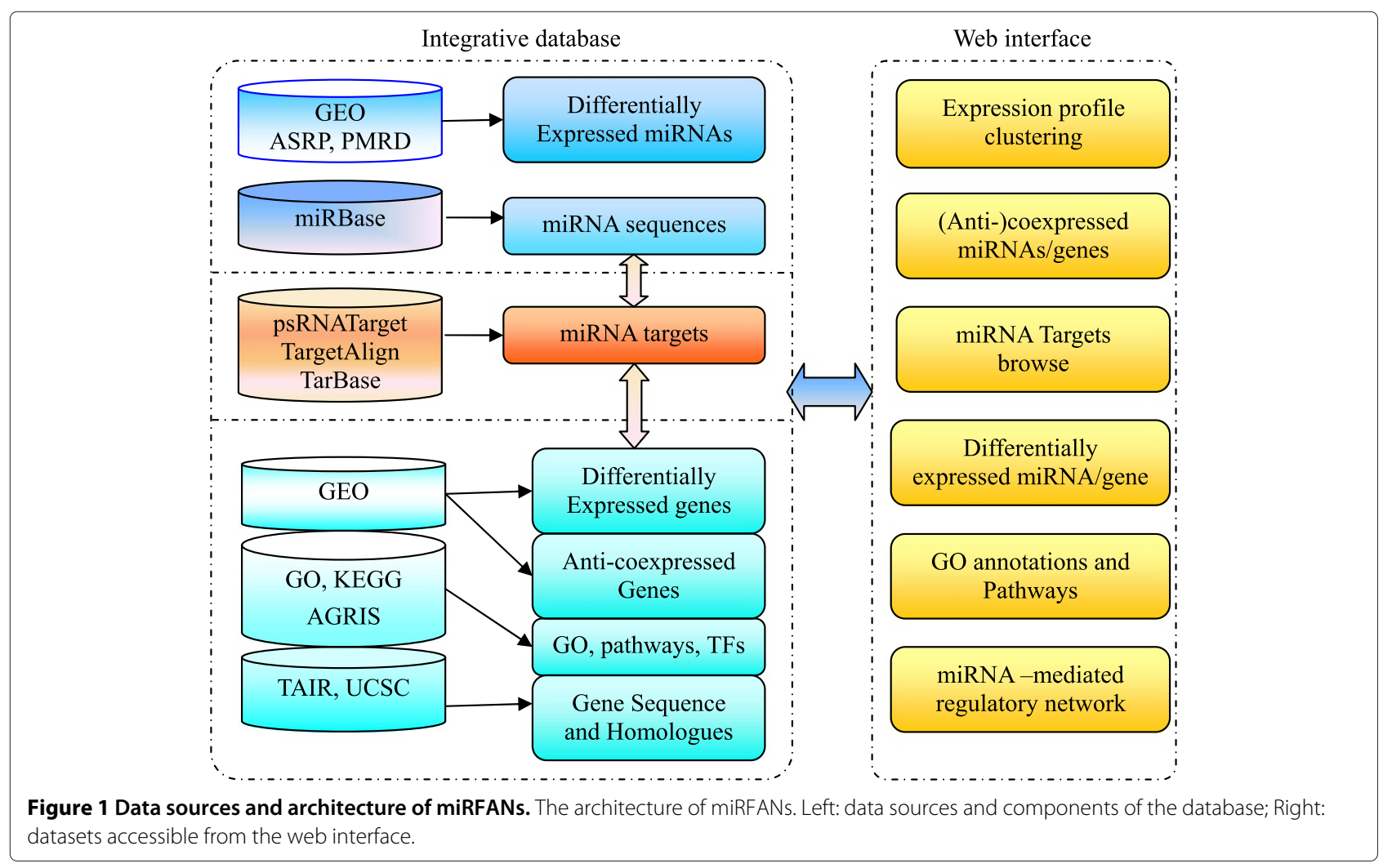


miRNAs and their targets under the same (or similar) experimental condition and in the same tissue, so that we can identify the specific spatio-temporal conditions under which the true regulation events happen. Specifically, we choose the experiments that measure expression profiles for both miRNAs and genes from the same samples, and also manually matched the expression profiles between miRNAs and their targets according to the experimental platform, plant growth and treatment condition and tissue. We then do differential expression comparison between miRNAs and their targets based on each pair of samples to check whether or not significantly up-regulated (or down-regulated) miRNAs lead to downregulation (or up-regulation) of the targets. This is helpful for us to reveal the tissues, environmental conditions in/under which miRNAs do significantly repress their targets, so as to deepen our understanding of the mechanism of miRNA regulation. Figure 2 illustrates the workflow of differential expression comparison.

\section{miRNA-mediated pathways}

In order to investigate the functional roles played by miRNAs, we integrate them into metabolic pathways to identify their effect on the downstream genes. As DIANAmirPath [34], the enrichment analysis of each pathway mediated by a miRNA is performed by Pearson's Chisquared test $\left(\chi^{2}=\sum\left((O-E)^{2} / E\right)\right)$, where $O$ (Observed) is the number of genes in the input dataset found to participate in a given pathway, and $E$ (Expected) is the number of genes expected by chance to be member of that pathway, given the pathway and input list size. The KEGG pathways involved by a miRNA of interest is ranked by the negative natural logarithm of the $p$-value $(-\ln p)$.

\section{miRNA-mediated genetic regulatory network}

Based on the miRNA-target and TF-target interactions, we extract a miRNA-mediated regulatory network according to the following rules: 1) all miRNA-target interactions recorded in TarBase and miRTarBase are included. 2) only TFs with more than $10 \mathrm{GO}$ terms are take into account so as to obtain an informative regulator network. 3) As miRNAs generated from the same miRNA family usually regulate common targets, miRNAs are represented by miRNA families so that the regulatory network is compact and can be well demonstrated via web-interface. As a result, we get a miRNA-mediated genetic regulatory network with 12 miRNA families, 41 TFs and 155 regulation relationship among them.

\section{Utility and Discussion}

\section{Web interface}

A user-friendly web interface is implemented to browse and analyze these data. Specifically, we develop two display modes, interactive table and summary text, to

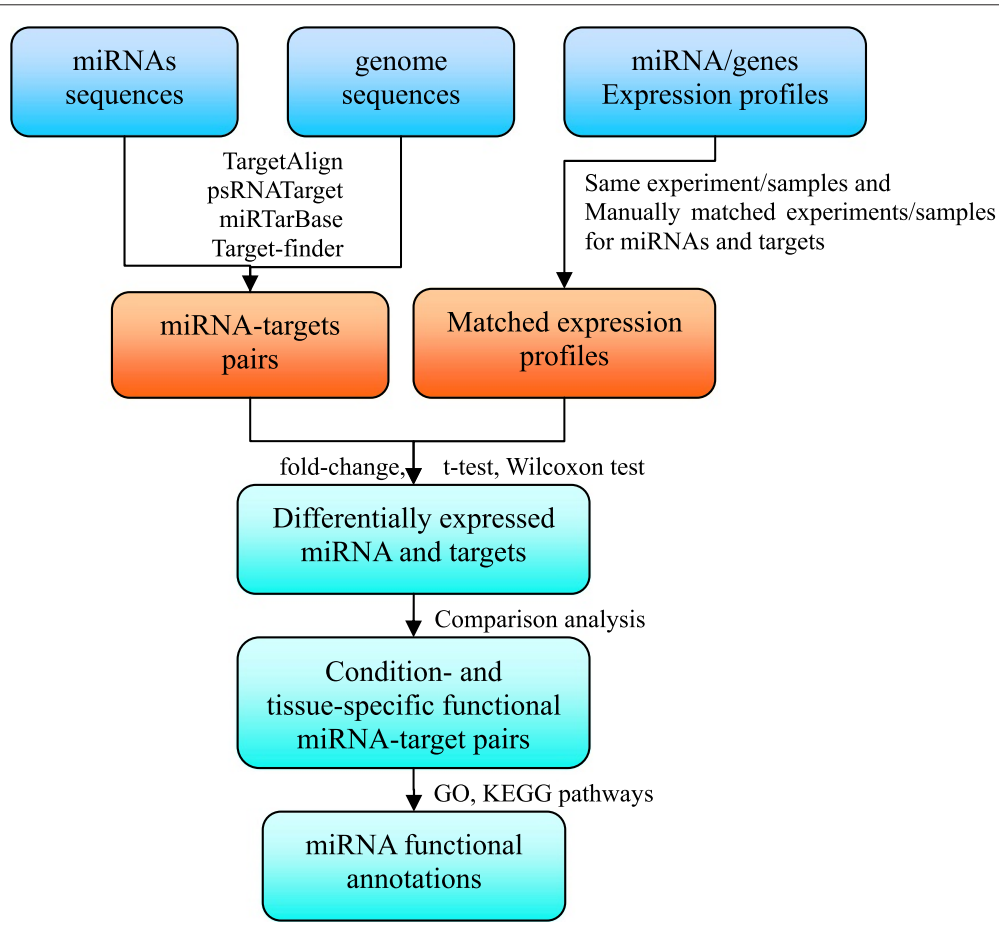

Figure 2 The workflow of differential expression comparison. The workflow of differential expression comparison between miRNAs and their targets, based on the samples of similar (or manually matched) environmental conditions and tissues. 
facilitate the data presentation and mining operators. In summary text mode, miRFANs shows summary information of a miRNA or a gene of interest in plain text, including the sequence, target genes, samples in which the miRNA or protein-coding gene is up-regulated and downregulated. Moreover, the TF family information, GO and pathway are also be shown for any protein-coding gene. In interactive table mode, all data are organized into tables. Each row of the table shows the number of target genes and samples with respect to a miRNA and a gene. Detail will be dynamically displayed when user click these numbers. Note that only in interactive table mode the analysis toolbox can be launched to carry out profiling, clustering, differential expressed analysis etc.

The database is developed using Microsoft SQL Server 2005 and consists of 19 physical tables (see Additional File 2: Description of the physical data model of the miRFANs database). The architecture of the server is designed by using Spring2.5+Struts2.0. The Web interface is developed by using XHTML, JSP, JavaScript, Jquery, Ajax and CSS. Both CSS and XHTML scripts respect the W3C standards and are validated by W3C online tools. Moreover, several visualization tools are employed to show the expression profiles, clustering, differential expressed comparison and miRNA-mediated genetic regulatory network. JHeatChart ${ }^{\mathrm{a}}$, a Java library for generating heat map charts is used to show the result of differential expressed analysis. Highcharts ${ }^{b}$ is a JavaScript charting library and is suitable for illustrating the expression profiles. we also exploit Java TreeView ${ }^{\mathrm{c}}$ to show the result of clustering and Cytoscape $\mathrm{Web}^{\mathrm{d}}$ to show miRNA-mediated genetic regulatory network.

\section{Search}

We develop a search module to support query for a miRNA or a coding gene of interest. For miRNAs, each of the two display modes mentioned above can be used to show the search result, and miRNA name (such as athmiR156a) or miRNA family name (such as ath-miR156 or miR156) can be taken as search keyword. When miRNA family name is input, the first matched miRNA of that miRNA family is used to guide the subsequent search process, and other miRNA members will also be listed as hyperlinks. For coding gene, only summary text display mode can be used at present and we will proceed to develop interactive table mode. For any miRNA, gene, GO term or metabolic pathway presented in the search result page, we set hypelinks to various well-known biology databases such as miRBase, TAIR, NCBI, KEGG.

\section{Analysis toolbox}

To facilitate the expression profiles analysis, we have developed an analysis toolbox that contains a number of classical expression profiles analysis and visualization functions. These functions include 1) identifying coexpressed (or anti-coexpressed) miRNAs/genes through computing Pearson correlation coefficients, Spearman correlation coefficients, cosine similarities and KL divergences based on the expression profiles of interest; 2) discovering differentially expressed miRNAs/genes via foldchange, t-test, Wilcoxon test, etc. 3) clustering analysis such as k-means, hierarchical clustering.

Figure 3(a) shows screenshots of the profiling module which is developed to browse and analyze the expression profiles by launching the Analysis toolbox. Figure 3(b) shows the screenshots of the functional annotation and pathway modules.

\section{Future work}

Further development of miRFANs will be mainly focused on at least three aspects. First, we plan to integrate miRNAs into transcriptional regulatory networks and thus construct miRNA-mediated regulatory networks for Arabidopsis thaliana. Second, we will include more analysis functions, such as building regulatory networks and genetic community discovery, into the analysis toolbox. Finally, we will continuously collect data and integrate other model plants into miRFANs.

\section{Conclusion}

miRFANs is an integrative database of Arabidopsis thaliana miRNAs and their target genes, expression profiles, function annotations and pathways. A friendly web interface is developed to browse and analyze of the data. We believe that miRFANs is a useful platform for exploring the regulatory functions of Arabidopsis thaliana miRNAs and can provide considerable value for many researchers.

\section{Availability and requirements}

miRFANs is freely available at http://www.cassavagenome.cn/mirfans. In the development of miRFANs, we follow the ordinary standards of web applications, and the Java scripts employed are cross-browser scripts. We have confirmed that miRFANs can work well with three webbrowsers, Google Chrome, Microsoft Internet Explorer and Mozilla Firefox, with no need for any plugins or special system requirements.

\section{Endnotes}

ahttp://www.javaheatmap.com/

bhttp://www.highcharts.com/

chttp://jtreeview.sourceforge.net/

${ }^{d}$ http://cytoscapeweb.cytoscape.org/ 


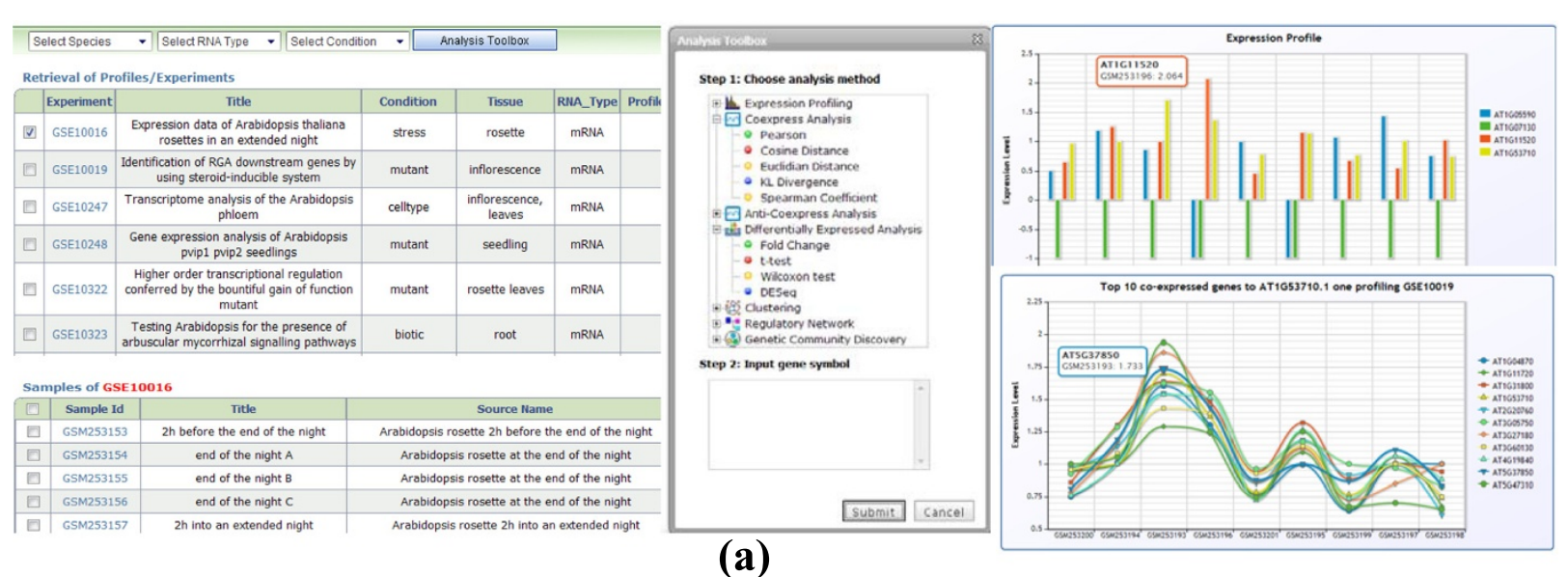

(a)
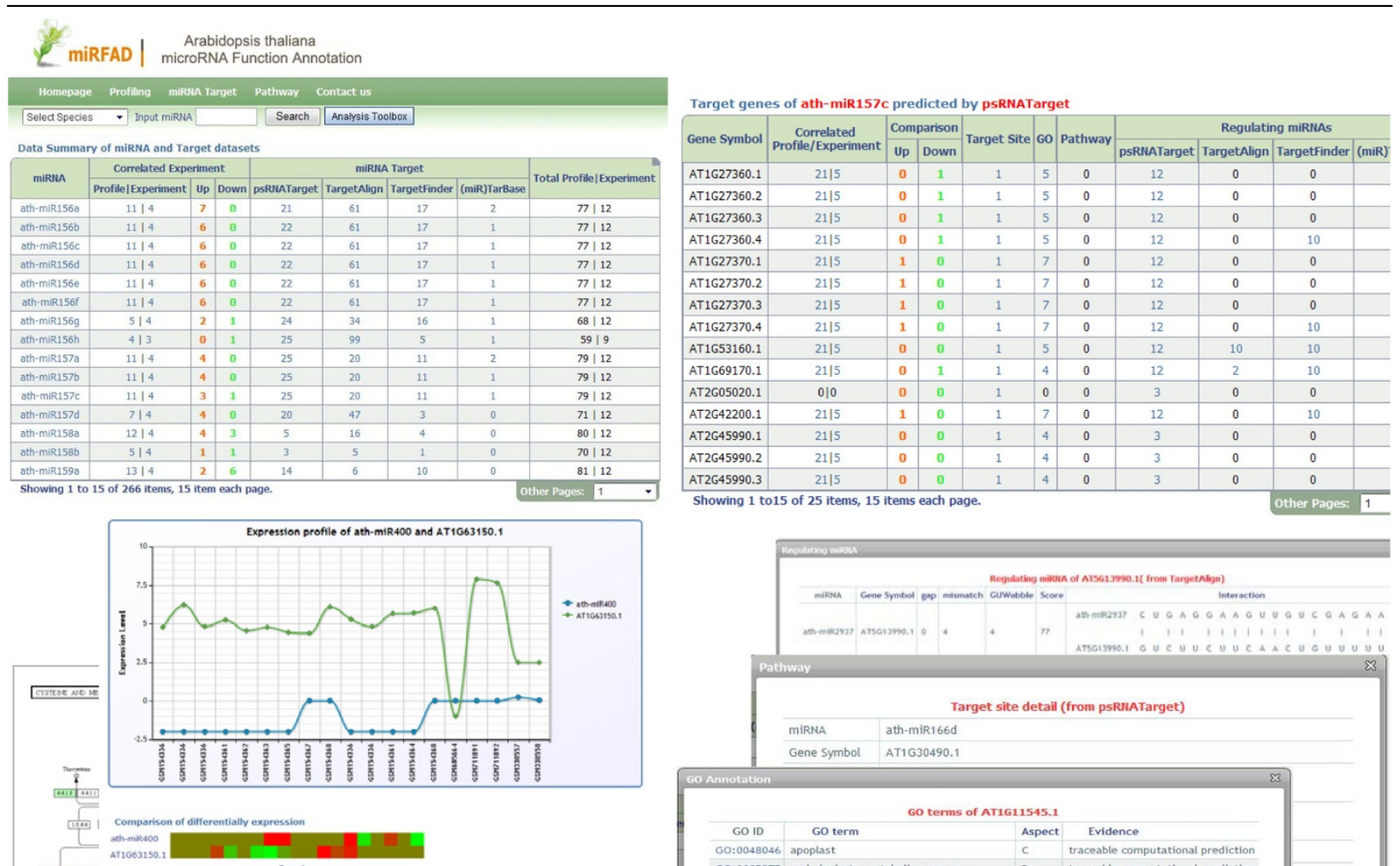

(2)

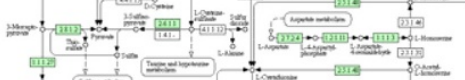

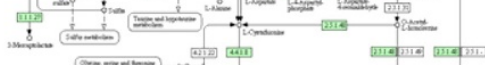

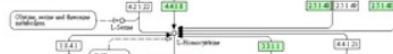

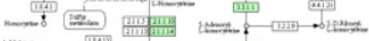

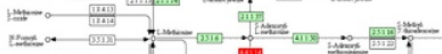

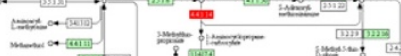

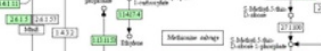

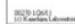

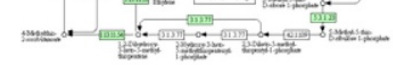

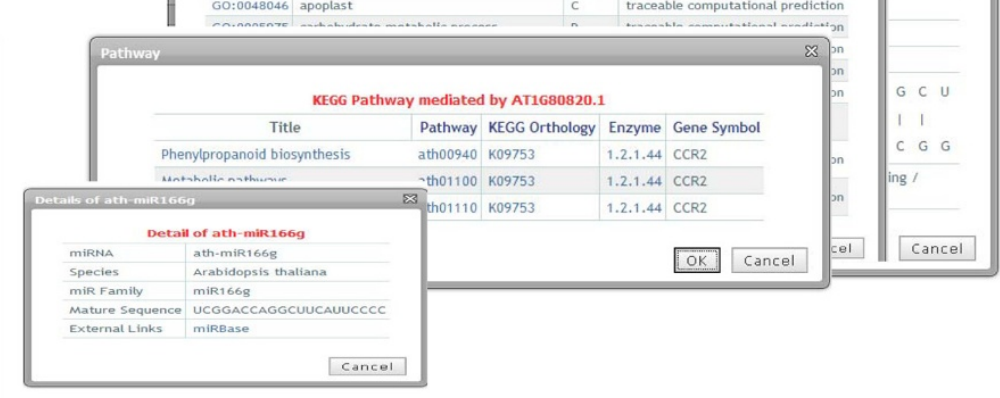

(b)

Figure 3 The screenshots of miRFANs. The screenshots of miRFANs. (a) Retrieval and statistical analysis of expression profiles for both miRNAs and target genes. Several types of expression analysis, including (anti-)coexpression, clustering, differential expression analysis, can be conducted by the toolkits. (b) Functional annotations of miRNAs by integrating the differential expression comparison and the GO as well as pathway terms. 


\section{Additional files}

Additional file 1: Sample additional file title. Detail of the datasets integrated into miRFANs database.

Additional file 2: Description of the relational database schema of miRFANs. The database is built on Microsoft SQL Server 2005 and includes 19 physical tables. The relational database schema is automatically generated using ModelRight 3.5 professional through reverse engineer from the database.

\section{Author's contributions}

$\mathrm{HL}$ designed the server and web interface, developed the profiling and miRNA target modules, and wrote the manuscript. TJ collected the GO and pathway data, developed the pathway module. LW and RL collected and preprocessed the expression data, and participated in the design of the relational database scheme. BX implemented the development of toolbox, and is also responsible for the update and maintenance of the database. SZ and JG provided support and participated discussions on the design and development of the database, coordinated the whole project and revised the manuscript.

\section{Acknowledgements}

This work was supported by National Basic Research Program of China under grant No. 2010CB227104, the National Natural Science Foundation of China under grants No. 31100954 , No. 61272380 and No. 61173118, the Open Research Fund Program of Shanghai Key Laboratory of Intelligent Information Processing, Fudan University (No. IIPL-2010-011), and PhD Program Scholarship Fund of Changzhou University (No. CCZUPY1001).

\section{Author details}

${ }^{1}$ Research Lab of Information Management, Changzhou University, Jiangsu, China. ${ }^{2}$ School of Computer Science, Fudan university, Shanghai, China. ${ }^{3}$ Department of Computer Science \& Technology, Tongji University, Shanghai, China. ${ }^{4}$ Shanghai Key Lab of Intelligent Information Processing, Fudan University, Shanghai, China. ${ }^{5}$ School of Information Science and Technology, Hainan University, Hainan, China.

Received: 18 October 2011 Accepted: 30 April 2012

Published: 14 May 2012

\section{References}

1. Bartel DP: MicroRNAs: genomics, biogenesis, mechanism, and function. Nucleic Acids Res 2004, 116:281-297.

2. Reinhart $B J$, Weinstein EG, Rhoades MW: MicroRNAs in plants. Genes Dev 2002, 16:1616-1626.

3. Lewis B, Burge C, Bartel D: Conserved seed pairing and often flanked by adenosines and indicates that thousands of human genes are microRNA targets. Cell 2005, 120:15-20.

4. Brodersen P, Sakvarelidze-Achard L, Bruun-Rasmussen M, Dunoyer P, Yamamoto YY, Sieburth L, Voinnet O: Widespread translational inhibition by plant miRNAs and siRNAs. Science 2008, 320:1185-1190.

5. Schwab R, Palatnik JF, Riester M, Schommer C, Schmid M, Weigel D: Specific effects of microRNAs on the plant transcriptome. Cell 2005, 8:517-527.

6. Mallory AC, Bartel DP, Bartel B: MicroRNA directed regulation of Arabidopsis AUXIN RESPONSE FACTOR17 is essential for proper development and modulates expression of early auxin response genes. Plant Cell 2005, 17:1360-1375.

7. Bartel B, Bartel DP: MicroRNAs: at the root of plant development. Plant Physiol 2003, 132:709-717.

8. Laufs P, Peaucelle MHA, Traas J: MicroRNA regulation of the CUC genes is required forboundary size control in Arabidopsis meristems. Development 2004, 131:4311-4322.

9. Mallory AC, Dugas DV, Bartel DP, Bartel B: MicroRNA regulation of NAC-domain targets is required for proper formation and separation of adjacent embryonic, vegetative, and floral organs. Curr Biol 2004, 14:1035-1046.
10. Sunkar R, Zhu JK: Novel and stress-regulated microRNAs and other small RNAs from Arabidopsis. Plant Cell 2004, 16:2001-2019.

11. Sunkar R: MicroRNAs with macro-effects on plant stress responses. Semin Cell Dev Biol 2010, 21(8):805-811.

12. Rhoades MW, Reinhart BJ, Lim LP, Burge CB, Bartel B, Bartel DP: Prediction of plant microRNA targets. Cell 2002, 110:513-520.

13. Rhee SY, Beavis W, Berardini TA, Chen G, Dixon D, Doyle A, Garcia-Hernandez M, Huala E, Lander G, Montoya M, Miller N, Mueller LA, Mundodi S, Reiser L, Tacklind J, Weems DC, Wu Y, Xu I, Yoo D, Yoon J, Zhang P: The Arabidopsis Information Resource (TAIR): a model organism database providing a centralized, curated gateway to Arabidopsis biology, research materials and community. NAR 2003, 31(1):224-228.

14. Kozomara A, S GJ: miRBase: integrating microRNA annotation and deep-sequencing data. NAR 2011, 39(Database Issue):D152-D157.

15. Gustafson AM, Allen E, Givan S, Smith D, Carrington JC, Kasschau KD ASRP: the Arabidopsis Small RNA Project Database. NAR 2005, 33(Database issue):D637-D640.

16. Zhang Z, Yu J, Li D, Zhang Z, Liu F, Zhou X, Wang T, Ling Y, Su Z: PMRD: plant microRNA database. NAR 2010, 38(Database issue):D806-D813.

17. Bielewicz D, Dolata J, Zielezinski A, Alaba S, Szarzynska B, Szczesniak MW Jarmolowski A, Szweykowska-Kulinska Z, Karlowski WM: mirEX: a platform for comparative exploration of plant pri-miRNA expression data. Nucl Acids Res 2012, 40(D1):D191-D197.

18. Szczesniak MW, Deorowicz S, Gapski J, Kaczynski L, Makalowska I: miRNEST database: an integrative approach in microRNA search and annotation. Nucleic Acids Res 2012, 40(D1):D198-D204.

19. Dai $X$, Zhao PX: psRNATarget: A Plant Small RNA Target Analysis Server. Nucleic Acids Res 2011, 39(suppl 2):W155-9.

20. Xie F, Zhang B: Target-align: a tool for plant microRNA target identification. Bioinformatics 2010, 26(23):3002-3003.

21. Moxon S, Schwach F, MacLean D: A toolkit for analysing large-scale plant small RNA datasets. Bioinformatics 2008, 24(19):2252-2253.

22. Hsu SD, Lin FM, Wu WY: miRTarBase: a database curates experimentally validated microRNA-target interactions. Nucleic Acids Res 2011, 39(suppl 1):D163-D169.

23. Papadopoulos GL, Reczko M, Simossis VA, Sethupathy P, Hatzigeorgiou AG: The database of experimentally supported targets: a functional update of TarBase. Nucleic Acids Res 2009, 37:D155-D158.

24. Edgar R, Domrachev M, E LA: Gene Expression Omnibus: NCBI gene expression and hybridization array data. Nucleic Acids Res 2002, 30:207-210.

25. Zhang Y: miRU: an automated plant miRNA target prediction server. Nucleic Acids Res 2005, 33(15):W701-W704.

26. Muckstein $\mathrm{U}$, Tafer $\mathrm{H}$, Hackermuller J: Thermodynamics of RNA-RNA binding. Bioinformatics 2006, 22:1177-1182.

27. Hackenberg M, Sturm M, Langenberger D: miRanalyzer: a microRNA detection and analysis tool for next-generation sequencing experiments. Nucleic Acids Res 2009, Suppl 2:W68-W76.

28. Langmead B, Trapnell C, Pop M, Salzberg SL: Ultrafast and memory-efficient alignment of short DNA sequences to the human genome. Genome Biology 2009, 10:R25.

29. Troyanskaya O, Cantor M, Sherlock G, Brown P, Hastie T, Tibshirani R, Botstein D, Altman RB: Missing value estimation methods for DNA microarrays. Bioinformatics 2001, 17:520-525.

30. Davuluri RV, Sun H, Palaniswamy SK, Matthews N, Molina C, Kurtz M, Grotewold E: AGRIS: Arabidopsis Gene Regulatory Information Server, an information resource of Arabidopsis cis-regulatory elements and transcription factors. BMC Bioinf 2003, 4:25.

31. Palaniswamy SK, James S, Sun H, Lamb RS, Davuluri RV, Grotewold E: AGRIS and AtRegNet: A platform to link cis-regulatory elements and transcription factors into regulatory networks. Plant Physiol 2006, 140:818-829.

32. Harris MA, Clark J, Ireland A, Lomax J, Ashburner M, Foulger R, Eilbeck K, Lewis S, Marshall B, Mungall C, Richter J, Rubin GM, Blake JA, Bult C, Dolan M, Drabkin H, Eppig JT, Hill DP, Ni L, Ringwald M, Balakrishnan R, Cherry JM, Christie KR, Costanzo MC, Dwight SS, Engel S, Fisk DG, 
Hirschman JE, Hong EL, Nash RS, Sethuraman A, Theesfeld CL, Botstein D, Dolinski K, Feierbach B, Berardini T, Mundodi S, Rhee SY, Apweiler R, Barrell D, Camon E, Dimmer E, Lee V, Chisholm R, Gaudet P, Kibbe W, Kishore R, Schwarz EM, Sternberg P, Gwinn M, Hannick L, Wortman J, Berriman M, Wood V, de la Cruz N, Tonellato P, Jaiswal P, Seigfried T, White R, Gene Ontology Consortium: The Gene Ontology (GO) database and informatics resource. Nucleic Acids Res 2004, 32:D258-D261.

33. Kanehisa M, Goto S: KEGG: kyoto encyclopedia of genes and genomes. Nucleic Acids Res 2000, 28:27-30.

34. Papadopoulos GL, Alexiou P, Maragkakis M, Reczko M, Hatzigeorgiou AG DIANA-mirPath: Integrating human and mouse microRNAs in pathways. Bioinformatics 2009, 25(15):1991-3.

doi:10.1186/1471-2229-12-68

Cite this article as: Liu et al: miRFANs: an integrated database for Arabidopsis thaliana microRNA function annotations. BMC Plant Biology 2012 12:68

Submit your next manuscript to BioMed Central and take full advantage of:

- Convenient online submission

- Thorough peer review

- No space constraints or color figure charges

- Immediate publication on acceptance

- Inclusion in PubMed, CAS, Scopus and Google Scholar

- Research which is freely available for redistribution

Submit your manuscript at www.biomedcentral.com/submit
() BioMed Central 